\title{
The SNFGTD method and its accuracy
}

\section{Bach, Henning}

\section{Published in:}

I E E E Transactions on Antennas and Propagation

Publication date:

1987

\section{Document Version}

Publisher's PDF, also known as Version of record

Link back to DTU Orbit

Citation (APA):

Bach, $\mathrm{H}$. (1987). The SNFGTD method and its accuracy. I E E E Transactions on Antennas and Propagation, 35(2), 169-175.

\section{General rights}

Copyright and moral rights for the publications made accessible in the public portal are retained by the authors and/or other copyright owners and it is a condition of accessing publications that users recognise and abide by the legal requirements associated with these rights.

- Users may download and print one copy of any publication from the public portal for the purpose of private study or research.

- You may not further distribute the material or use it for any profit-making activity or commercial gain

- You may freely distribute the URL identifying the publication in the public portal

If you believe that this document breaches copyright please contact us providing details, and we will remove access to the work immediately and investigate your claim. 


\title{
The SNFGTD Method and Its Accuracy
}

\author{
HENNING BACH AND HANS-HENRIK VISKUM, MEMBER, IEEE
}

\begin{abstract}
The spherical near-field geometrical theory of diffraction (SNFGTD) method is an extended aperture method by which the near field from an antenna is computed on a spherical surface enclosing the antenna using the geometrical theory of diffraction. The far field is subsequently found by means of a spherical near-field to far-field transformation based on a spherical wave expansion of the near field. Due to the properties of the SNF-transformation, the total far field may be obtained as a sum of transformed contributions which facilitates analysis of collimated beams. It is demonstrated that the method possesses some advantages over traditional methods of pattern prediction, but also that the accuracy of the method is determined by the quasioptical methods used to calculate the near field.
\end{abstract}

\section{INTRODUCTION}

$\mathrm{T}$ HE TWO CLASSICAL methods for the determination of fields radiated by reflector antennas, the current integration method (CIM) and the aperture integral method (AIM), are based on the induction and equivalence theorems of electromagnetic theory, respectively. The two methods are illustrated in Fig. 1(a). By the induction method, the scattered field $E_{s}$ is found from the induced currents on the scatterer, here called the reflector, and the total field $E$ is given by

$$
E=E_{i}+E_{s}
$$

where $E_{i}$ is the free space field from the primary field source.

By the equivalence method, the radiated field $E$ is found from the total tangential field $E_{l}$ on a closed surface, here called the extended aperture, enclosing the antenna completely. In both cases, the resulting fields are expressed through complicated surface integrals; since these can always in principle be calculated, the original problems are reduced to the determination of the surface current distribution on the reflector and the tangential field distribution on the aperture surface. It is worthwhile to note that the above methods are exact methods. This means that if the exact current distribution and the exact aperture distribution are known, the two methods yield the same exact result.

In practical applications, the surfaces are usually chosen as shown in Fig. 1(b). In the classical CIM, the integration is carried out only over the illuminated part of the reflector. This is equivalent to assuming that the current on the back of the reflector is zero. Likewise, in the classical AIM, the field is found from the tangential component of the scattered field, and

Manuscript received May 21, 1986; revised July 1, 1986. This work was supported by the European Space Research and Technology Centre.

H. Bach is with the Electromagnetics Institute, Technical University of Denmark, DK-2800 Lyngby, Denmark.

H.-H. Viskum is with INTELSAT, 3400 International Drive, Washington, DC 20008

IEEE Log Number 8612256.
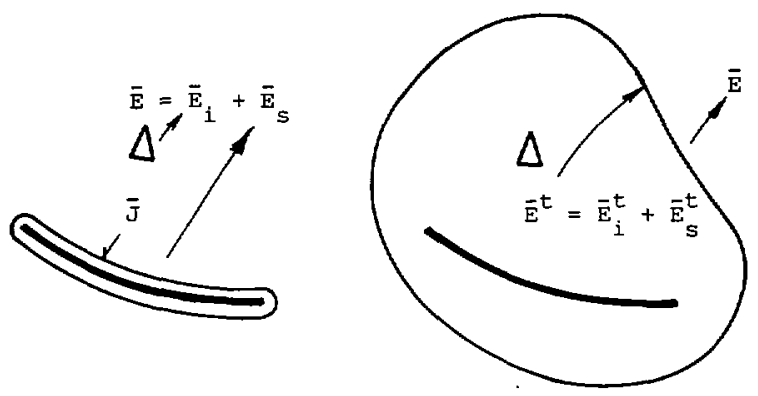

(a)
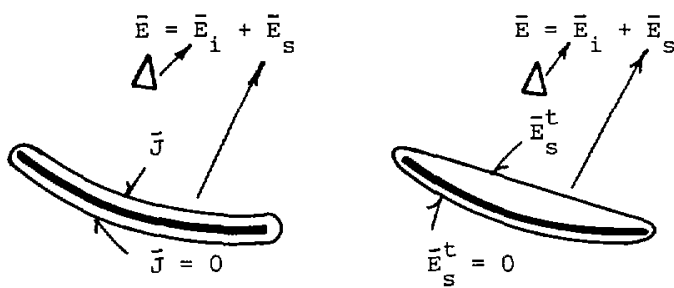

(b)
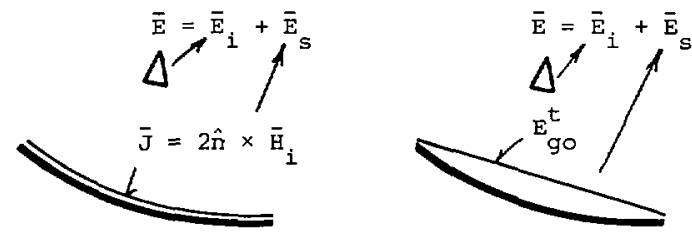

(c)

Fig. 1. Pattern predictions methods. The current integration method (left), the aperture integral method (right).

the integration is done over only that part of the extended aperture that caps the reflector. This is equivalent to assuming a zero tangential component of the scattered field on the back of the reflector. Recently, it has been shown [1] that provided the exact current and aperture distributions are used, the two methods, also in this case, yield identical results. It is interesting, however, that the field thus determined is not the exact field from the antenna since by both methods the contributions from the back side of the reflector are neglected.

In Fig. 1(c) are shown the approximations used in practical applications. In the CIM, the surface current is approximated by the physical optics current $J=2 \hat{n} \times H_{i}$ and similarly, in the AIM, the aperture field is approximated by the geometrical optics field. In [1], it has also been shown that the CIM with the physical optics (PO) approximation and the AIM with the geometrical optics approximation yield equal copolar fields within these approximations provided the aperture caps the reflector. Since this is the case in most practical applications, only the current integration method with the physical optics approximation is considered. Furthermore, the 
discussion is limited to directions close to the main lobe $(\theta<$ $18^{\circ}$ ) where PO is known to yield accurate results [1].

\section{The SNFGTD Method}

A method of exact analysis may be based upon the surface integral equation formulation and the moment method (MM) which implies a direct numerical determination of the current distribution on the reflector. The moment method in its various forms is known to yield very accurate results but the applicability of the method is limited by the fact that the technique involves numerical solution of large systems of linear equations, the size of which increases rapidly with the reflector diameter. Thus moment method solutions may be used for purposes of comparison in special cases [7] but the technique must be excluded as a practicable method for analysis of reflector antennas.

An alternative method for accurate analysis may be based on the equivalence theorem if the total tangential field can be obtained on the extended aperture. By the spherical near-field geometrical theory of diffraction (SNFGTD) method, the total tangential field, using geometrical theory of diffraction, is computed over a spherical surface referred to as the near-field sphere enclosing the antenna. Subsequently the far field is determined by a spherical wave expansion of the near field. The method was originally used by Jensen and Larsen [2] and has been later described in [3]-[5], so only a short review will be presented here. Recently the method has also been considered in [6] and [7].

It should be noted that other extended aperture methods, related to the planar and cylindrical near-field testing techniques, are in use. In both of these techniques, the extended aperture surface, in contrast to the spherical case, extends to infinity, which implies that the surface must be truncated prior to the far-field transformation. This of course is a source of errors.

\section{A. The Near-Field Calculation}

The tangential components of the field on the near-field sphere may be conveniently obtained using the geometrical theory of diffraction (GTD) but in principle; any adequate method may, of course, be used. Thus the computation of the near field is a standard task since well-known and wellestablished techniques reported in the open literature may be used. Such calculations are given in [10] and [11], for example. In the present paper, the uniform asymptotic formulation of the geometrical theory of diffraction by Kouyoumjian is used and in particular, the diffraction coefficients given in [12]. Also, the theory of slope diffraction as presented in [13] and the theory of equivalent currents given in [14] are used.

In Fig. 2 is shown a simple focused parabolic reflector antenna and the near field sphere with radius $r_{0}$ centered at the focus of the antenna. Since the field is computed close to the antenna, only one reflected ray passes through any field point $F$ such that the geometrical optics caustic encountered in the main beam direction is avoided. In all cases presented in this paper the following contributions, as indicated in Fig. 2, are included: direct and reflected rays, singly and doubly dif-

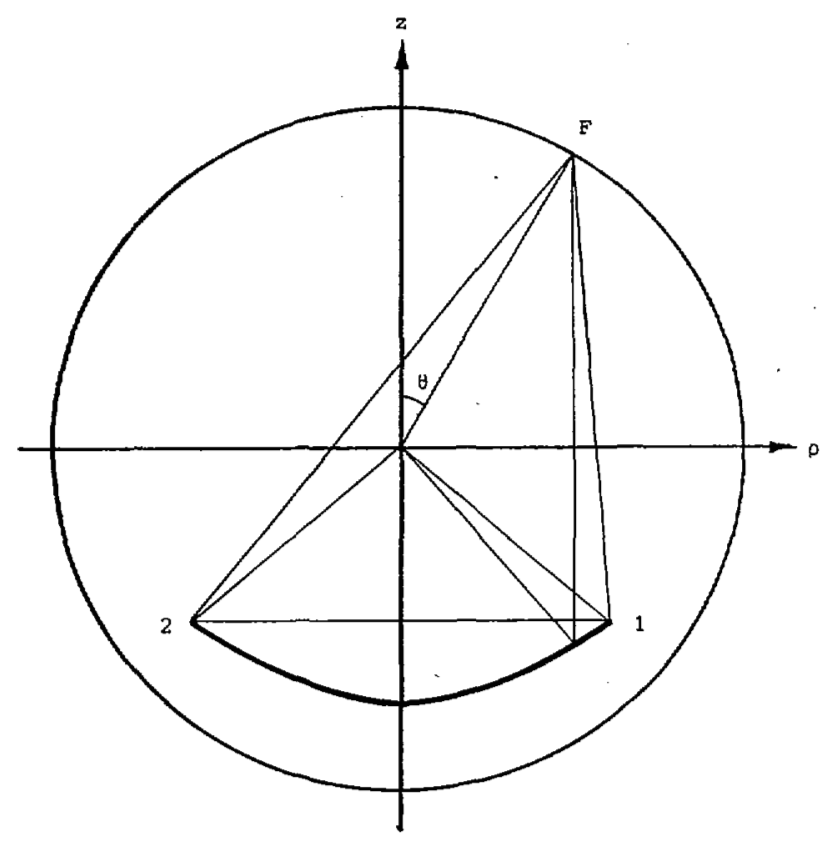

Fig. 2. Parabolic reflector antennas with near-field sphere.

fracted rays from edges 1 and 2 , slope diffraction contribution as well as a correction term for the caustic formed by the diffracted fields on the axis of the antenna. Thus higher order edge diffracted rays and all surface diffracted rays are neglected. This effect will be commented on later in the paper.

\section{B. Spherical Near-Field to Far-Field Transformation}

The SNF-transformation algorithm is based on the following formulas for a spherical vector wave expansion:

$$
\bar{E}(\theta, \Phi)=\sum_{s, m, n} Q_{s m n} \bar{F}_{s m n}(r=\infty, \theta, \Phi)
$$

and

$$
Q_{s m n}=\int_{0}^{2 \pi} \int_{0}^{\pi} \hat{r} \cdot\left(\bar{E}\left(r_{0}, \theta, \phi\right) \times \bar{F}_{s m n}\left(r_{0}, \theta, \Phi\right) \sin \theta\right) d \theta d \Phi .
$$

Here $\bar{F}_{s m n}$ denotes the spherical vector wave functions while $\bar{E}(\theta, \Phi)$ denotes the wanted far-field patterns and $\bar{E}\left(r_{0}, \theta, \Phi\right)$ the field on the near-field sphere of radius $r_{0}$. Due to the factor $\hat{r}$ in the integrand of the expansion coefficients $Q_{s m n}$, only the tangential field contributes to the integral. Furthermore, as a matter of curiosity, it may be noticed that due to the factor $\sin$ $\theta$ in the same integral, the on-axis near field value does not contribute to the far field. This illustrates the fact that the SNF-transformation does not establish a one-to-one correspondence between points on the near-field and the far-field spheres.

The reformulation of the above formulas into discrete formulas which may serve as the basis of efficient computer programs is described in [8], [9], and [15]. The resulting formulas are somewhat complicated and will not be repeated here since for our purpose it suffices to state that they express the far field as a complicated superposition of spherical wave functions. We shall, however, emphasize two important properties of the transformation formula, namely that the 
formula is linear and that all summations in the formula are finite. This implies that the indices $n$ and $m$ are limited to maximum values $n_{\max }$ and $m_{\max }$ depending on the structure of the near field. As we shall show later, these properties may be utilized to provide new insight in the structure of collimated fields.

Since the SNFGTD is not based on the usual approximations mentioned in the introduction, it offers a possibility of comparison of results in addition to the MM method also within the main beam region. Furthermore, it should be noted that the method yields the field from the antenna at any distance and in any direction without using different methods in various regions around the antenna.

A large amount of analysis, including defocused and offset reflector antennas, has been carried out but to keep the present paper concise, the subsequent discussion will be based on results obtained for a $20 \lambda$ diameter, rotational symmetric, focused parabolic reflector antenna fed by either a Hertzian dipole or a Huygens source both polarized in the $x$-direction on the $z$-axis. These simple sources were chosen to avoid errors related to non-Maxwellian source-field functions, while the relatively small diameter was chosen to be able to produce an MM solution for reference purposes. The $f / d$ ratio of the antenna considered is 0.4 and the rim subtends a half-angle of $64^{\circ}$ when seen from the feed. This provides an edge taper of $-7.12 \mathrm{~dB}$ in the $E$-plane of the dipole and $-2.86 \mathrm{~dB}$ for the Huygens source. For both configurations the $E$-plane field, the $H$-plane field and the maximum cross polar field were computed. Following Ludwig's third definition [16] the maximum cross polar radiation appears in the $\Phi=45^{\circ}$ planes. The radius of the near-field sphere, which was centered at the focus of the antenna, in all cases was $16 \lambda$.

\section{The SNF-Transforms}

The general relation between the far-field pattern $E(\theta, \Phi)$ and the near-field pattern $E_{n}\left(r_{0}, \theta, \Phi\right)$ on the near-field sphere may in symbolic form be written

$$
E=\operatorname{SNF}\left(E_{n}\right)
$$

where the operator SNF denotes the near-field to far-field transformation. As mentioned above, the SNF-transform is linear and bandlimited, i.e., all summations in the discrete transformation formula are finite. Thus, if the near field is divided into two arbitrary constituents $E_{1}$ and $E_{2}$, i.e.,

$$
E_{n}=E_{1}+E_{2}
$$

then

$$
E=\operatorname{SNF}\left(E_{n}\right)=\operatorname{SNF}\left(E_{1}\right)+\operatorname{SNF}\left(E_{2}\right)
$$

which implies that the far-field pattern is the sum of the SNF transforms of the near-field constituents. When the field constituents $E_{1}$ and $E_{2}$ are physical fields which may be represented by the same number of modes $n_{\max }, m_{\max }$ as the original field $E$, the SNF-transforms of $E_{1}$ and $E_{2}$ are unique and thus independent of the radius of the near-field sphere.

The above result may be applied to reflector antenna analysis since using GTD to compute the near field it may, for example, be expressed through

$$
E_{n}=E_{\mathrm{dir}}+E_{\mathrm{ref}}+E_{\mathrm{dif}}+E_{\text {sld }}
$$

where $E_{\mathrm{dir}}$ is the direct field from the feed horn, $E_{\text {ref }}$ the reflected field, $E_{\text {dif }}$ the diffracted field and $E_{\text {sld }}$ the slope diffracted field. Separate transformation of each individual field constituent then yields the result

$$
\begin{aligned}
E=\operatorname{SNF}\left(E_{n}\right)=\operatorname{SNF}\left(E_{\mathrm{dir}}\right)+\operatorname{SNF} & \left(E_{\mathrm{ref}}\right) \\
& +\operatorname{SNF}\left(E_{\mathrm{dif}}\right)+\operatorname{SNF}\left(E_{\mathrm{sld}}\right)
\end{aligned}
$$

which shows that the far field may be obtained as the sum of the SNF-transforms of the near-field constituents corresponding to each individual ray system. It is important to note that in this case the near field constituents are not bandlimited, since they possess strong discontinuities at the shadow boundaries of the various ray systems and thus do not represent physical fields. It turns out, however, that provided the same number of modes, $n_{\max }$ and $m_{\max }$, necessary to represent the total field $E$, is also used when transforming the various ray systems the dependence of the SNF-transforms on the radius of the nearfield sphere is weak. Thus it is possible to associate a single SNF-transform with each ray system.

In Fig. 3, the SNF-transforms of the direct, the reflected, the diffracted and the slope diffracted fields are shown together with the total far field for the two different excitations of the reflector antenna in question. For dipole excitation, it appears that the average sidelobe level is determined by the direct field while this is very low in the Huygens case. Apart from this, the sidelobes are determined by the reflected and the diffracted fields. For both excitations, the reflected field dominates over the diffracted field contributions in the near-in sidelobes while the roles are interchanged in the outer sidelobes. Slope diffraction contributes very little to the sidelobes, in particular in the $H$-plane where the edge taper is zero. The bottom plots show that the structure of the cross polar fields in the two cases is very different. Firstly, the level of the first cross polar lobe in the dipole case is determined solely by the reflected field while in the Huygens case, the diffracted field yields a significant contribution to the cross polar level. In both cases, the diffracted fields dominate over the reflected field in the outer lobes. It may be demonstrated that higher order diffracted fields do not contribute significantly to the field for which reason they have been neglected.

It is a characteristic feature of the geometrical theory of diffraction that the total field is obtained as a sum of ray contributions which originate from specific, well defined, parts of the scatterer in question. This property of the GTD often leads to a better physical understanding of the scattering process under consideration. By the SNFGTD technique, the total field is similarly obtained as a sum of transformed contributions which may be associated with specific, well defined, parts of the scattering structure so that the above mentioned feature of the GTD is retained even within the collimated region of the main beam of the antenna. As seen from the above example, this property of the SNFGTDmethod may yield a deeper insight into the structure of collimated fields, a property which will be used in the 


\section{Parabolic Reflector Antenna}

$$
D=20 \lambda, F / D=0.4 \text {, }
$$
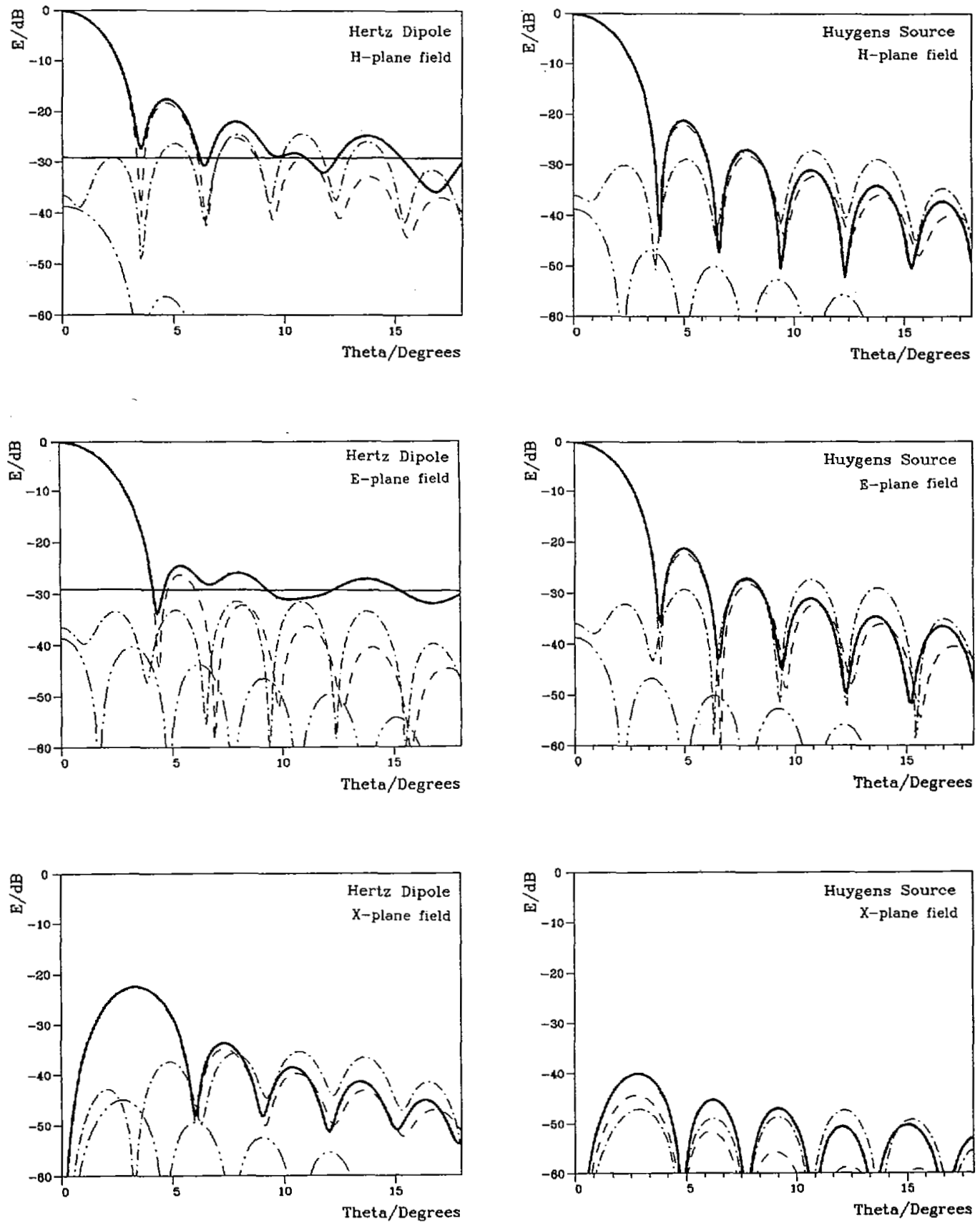

Fig. 3. SNF-transforms of direct field $(-)$, reflected field (---), diffracted field $(-\cdot-)$ and slope diffracted field $(-\cdots-)$. The heavy solid line is the total field. Dipole excitation (left), Huygens excitation (right).

following discussion to assess the accuracy of the SNFGTD method.

\section{Comparison of Results}

In this section the SNFGTD results for the above mentioned reflector antenna are compared to physical optics solutions and moment method solutions. The PO computation was done using the general reflector antenna analysis and synthesis program (GRASP) [17], while the MM solution is based on a special high accuracy moment method program version [18]. Table I shows that the directivities computed by the three methods differ only by a few hundredths of a $\mathrm{dB}$. Thus the comparisons shown in the plot are absolute comparisons.

In the first case, Fig. 4(a), for dipole excitation it is noted that the $H$-plane amplitude and phase pattern are practically identical, within the accuracy of the plots, for all three methods. This fact was reported already in [4] and repeated in [7]. In the $E$-plane, a similar agreement is obtained except at, . 
TABLE I

DIRECTIVITIES IN dB FOR $20 \lambda$ REFLECTOR ANTENNA

\begin{tabular}{lccc} 
& SNFGTD & MM & PO \\
Hertzian dipole & 30.76 & 30.79 & 30.82 \\
Huygens source & 33.81 & 33.87 & 33.79 \\
\hline
\end{tabular}

\section{Parabolic Reflector Antenna \\ $D=20 \lambda, F / D=0.4$, Dipole Source}
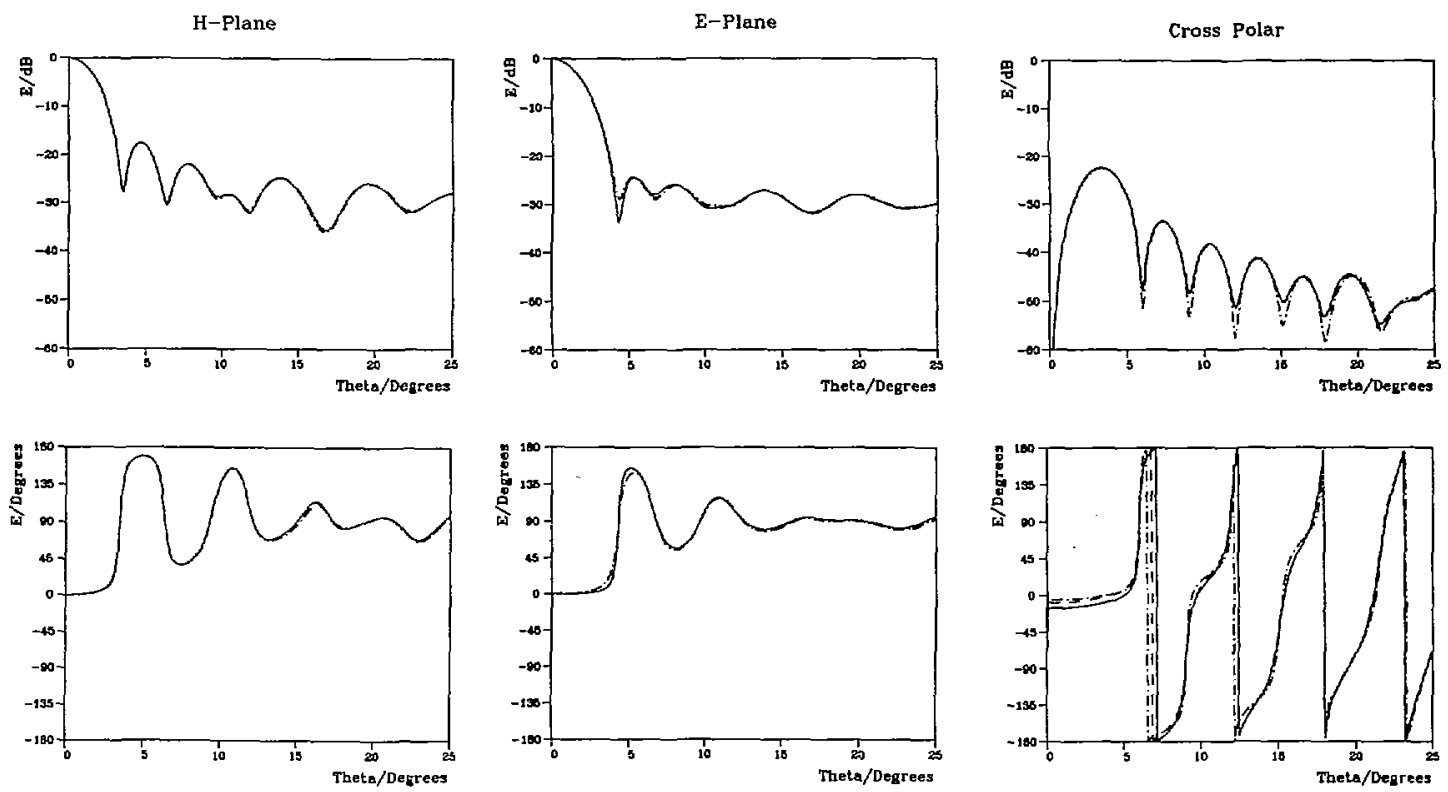

\section{Parabolic Reflector Antenna \\ $D=20 \lambda, F / D=0.4$, Huygens Source}

H-Plane
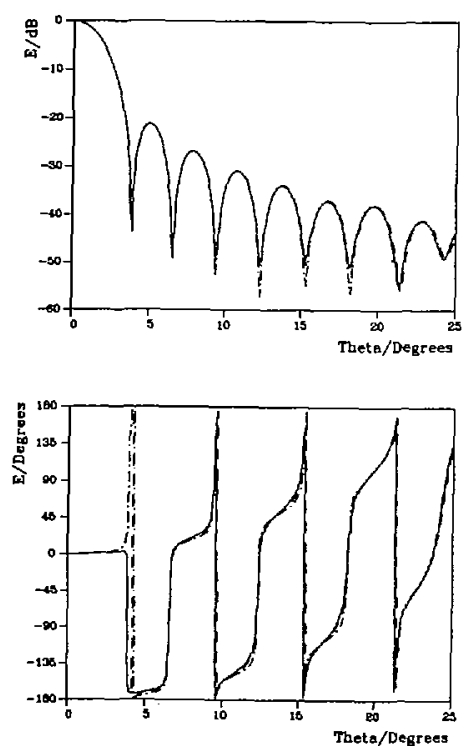

E-Plane
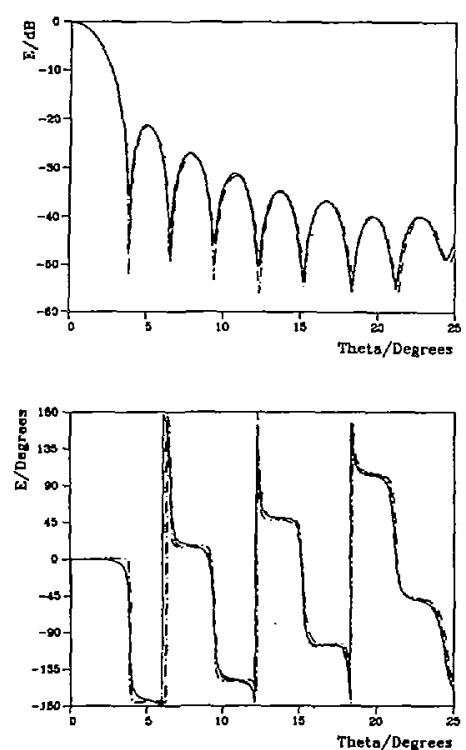

Cross Polar
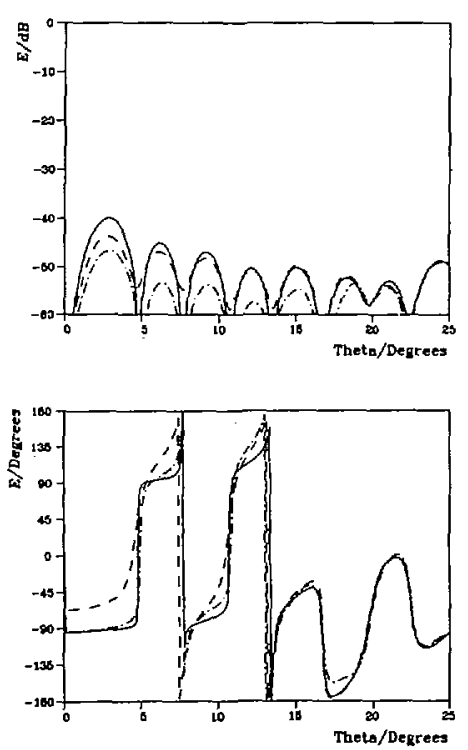

Fig. 4. Comparisons between SNFGTD (-), MM (--) and PO (-·-·). Dipole excitation (top), Huygens source (bottom). 
and close to, the minimum next to the main beam. Here the SNFGTD predicts a somewhat deeper minimum than the two other methods, and the SNFGTD phase pattern also differs slightly in this region. The cross polar patterns agree well but the deviations nevertheless are more significant, in particular close to the minima. However, it is seen that the same level of the first cross polar lobe is predicted by all three methods.

In Fig. 4(b), similar computations are presented for the Huygens source excitation. Also in this case the $H$-plane patterns are almost identical for the three methods although some minor deviations are seen. In the $E$-plane, again discrepancies are present close to the minima so the overall accuracy has been reduced for both patterns. This fact becomes clear when the cross polar fields are considered since here the three methods differ considerably both in amplitude and phase such that the level of the first cross polar lobe is now predicted differently by the three methods. The highest level is predicted by the SNFGTD and the lowest by PO while in the outer lobes the SNFGTD and MM results are in good agreement.

As mentioned above, the AIM is in principle an exact method, so the observed differences between the MM and the SNFGTD results must originate from errors in the GTD near field on the spherical aperture surface. To investigate this, the MM solution was transformed from the far-field to near-field sphere and compared with the GTD near field pattern. This revealed that discrepancies occurred in the regions close to the geometrical optics shadow boundaries so the error in the cross polar level seemed to be caused by inaccuracies in the diffraction coefficients used to calculate the field close to the shadow boundaries. To check this hypothesis, the GTD near-field data in the region close to the reflection boundary were substituted by the corresponding MM data and the resulting "hybrid" near field was transformed to the far field. In Fig. 5 is shown a comparison between the hybrid GTD-MM far field, the original far field and the PO solution. It appears that an almost complete agreement between the hybrid data and the MM data is now obtained which confirms the above hypothesis. This is an interesting result since it explains why the SNFGTD results differ from the MM results mainly on the first cross polar lobe. In the main beam direction, the contributing diffracted fields are radiated close to the reflection boundary while this is not the case in the sidelobe regions.

\section{CONCLUSION}

The SNFGTD method has been discussed and related to other pattern prediction methods for reflector antennas. It has been demonstrated that by the SNFGTD method, a deeper insight into the structure of collimated fields may be obtained since the total field is obtained as a sum of transformed fields which may be associated with specific, well defined parts of the antenna under consideration. Furthermore, it has been shown that for the reflector antenna in question, the three methods, PO, MM, and SNFGTD yield practically identical results in and close to the main lobe as far as the co-polar patterns are concerned while for the cross polar components, certain discrepancies were observed. The discrepancies be-
Parabolic Reflector Antenna $\mathrm{D}=20 \lambda, F / D=0.4$. Huygens Source
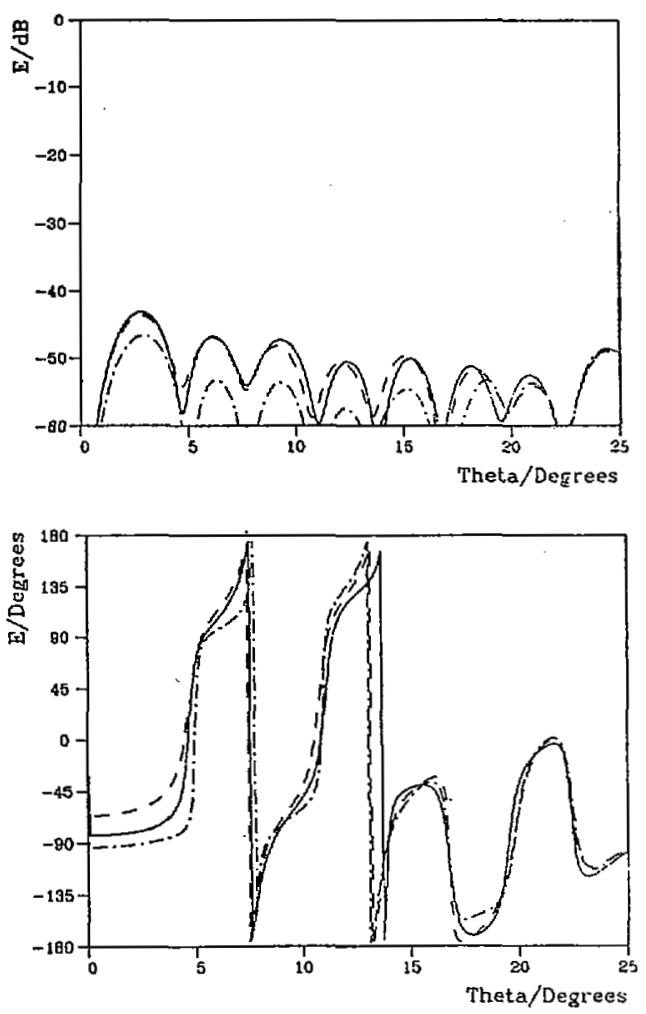

Cross Polar

Fig. 5. Comparison between hybrid SNFGTD-MM data (-), MM data $(--)$ and PO data $(-\cdot-\cdot)$.

tween the SNFGTD results and the MM results were shown to be related to inaccuracies in the field computations close to the shadow boundaries. It should be noted that the PO results which may not be correct anywhere, even within the main beam, probably could be improved considerably by inclusion of the fringe wave fields [19], [20].

\section{ACKNOWLEDGMENT}

The authors are indebted to Dr. F. H. Larsen and Mr. G. Hau Lemanczyk, Electromagnetics Institute and Dr. A. Frandsen, TICRA A/S for their interest and help, which contributed to this work. The authors also want to thank Dr. Arthur Yaghjian for valuable comments.

\section{REFERENCES}

[I] A. D. Yaghjian, "Equivalence of surface current and aperture field integrations for reflector antennas," IEEE Trans. Antennas Propagat., vol. AP-32, no. 12, pp. 1355-1358, Dec. 1984.

[2] F. Jensen and F. H. Larsen, "Spherical near-field technique," presented at IEEE Antennas Propagat. Soc. Int. Symp.. Univ. Stanford, 1977.

[3] H. Bach, "Field computations for reflector antennas by GTD," Electromagn. Inst. Tech. Univ. Denmark, 1979, NB 122.

[4] H. Bach, A. Frandsen, and F. H. Larsen, "Pattern prediction for a focused reflector antenna using GTD and near-field to far-field transformations," presented at Second. Int. Conf. Antennas Propagat., Univ. York, 1981.

[5] H.-H. Viskum, "Pattern prediction for a defocused reflector antenna using GTD and near-field to far-field transformation," presented at Third Int. Conf. Antennas Propagat., Univ. East Anglia, 1983. 
[6] M. S. Narasimhan and S. Christopher, "A new method of analysis of the near and far fields of paraboloidal reflectors," IEEE Trans. Antennas Propagat., vol. AP-32, no. 1, pp. 13-19, Jan. 1984.

[7] H. Bach, "Comments on "A new method of analysis of the near and far fields of parabolic reflector antennas'," IEEE Trans. Antennas Propagat., vol. AP-33, no. 1, pp. 116-117, Jan. 1985.

[8] F. Jensen, "On the probe compensation for near-field measurements on a sphere," Archiv Elekt. Ubertragung., vol. 29, no. 7/8, pp. 305308, July/Aug. 1975.

[9] F. H. Larsen, "Probe correction of spherical near-field measurements," Electron. Lett., vol. 13, no. 14, pp. 393-395, July 1977.

[10] T. E. Cherot, "Calculation of near field of circular aperture antenna using geometrical theory of diffraction," IEEE Trans. Electromagn. Compat., vol. EMC-13, no. 2, pp. 29-34, May 1971.

[11] M. S. Narasimhan and K. M. Prasad, "GTD analysis of the near-field patterns of a prime-focus symmetric paraboloidal reflector antenna," IEEE Trans. Antennas Propagat., vol. AP-29, no. 6, pp. 959-961, Nov. 1981.

[12] R. G. Kouyoumjian and P. H. Pathak, "A uniform geometrical theory of diffraction for an edge in a perfectly conducting surface," Proc. IEEE, vol. 62, no. 11, pp. 1448-1461, Nov. 1974.

[13] N. C. Albertsen, H. Bach, P. Balling, O. Sorensen and K. Pontoppidan, "Radiation pattern prediction for high frequency antennas," ESTEC Contract 2866/76. Electromagn. Inst., Tech. Univ. Denmark, Final Rep., June 1977.

[14] C. E. Ryan, Jr., and L. Peters, Jr., "Evaluation of edge-diffracted fields including equivalent currents for the caustic regions," IEEE Trans. Antennas Propagat., vol. AP-17, pp. 292-299, May 1969.

[15] P. F. Wacker, "Near-field antenna measurements using a spherical scan: efficient data reduction with probe correction," Inst. Elec. Eng. Conf. Pub. 113, Conf. Precision Electromagn. Meas.

[16] A. C. Ludwig, "The definition of cross polarization," IEEE Trans. Antennas Propagat., vol. AP-21, pp. 116-119, Jan. 1973.

[17] R. Jørgensen, "Manual for GRASP6," Rep. S-175-06, TICRA A/S, Copenhagen, 1983.

[18] Rydahl, ROT2X, Users Guide, IR 130, Electromagn. Inst. Tech. Univ. Denmark, Sept. 1977.

[19] P. Y. Ufimtsev, "Method of edge waves in the physical theory of diffraction, (from Russian "Method krayevykh voln $v$ fizicheskoy teorii difraktsii" Izd-vo Sov. Radio, pp. 1-243, 1962) translation prepared by the U.S. Air Force Foreign Technol. Div., WrightPatterson AFB, OH, released for public distribution Sept. 7, 1971

[20] H. Bach, "A note on antennas, definitions and methods." IEEE Trans. Antennas Propagat., to be published.

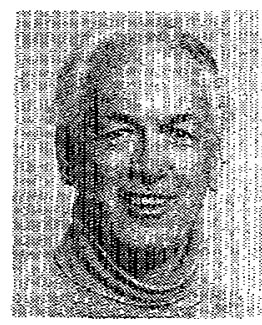

Henning Bach was born in Denmark on October 9, 1929. He received the M.Sc. and Ph.D. degrees in electrical engineering from the Technical University of Denmark in 1962 and 1968, respectively.

He was with the Electromagnetics Institute, Technical University of Denmark from 1962-1967. In 1967-1968 he spent a year at Arecibo Observatory, Cornell University, Ithaca, NY, as a member of the ionosphere group. From 1968 to the present he has been with the Electromagnetics Institute, Technical University of Denmark, apart from 1979-1980, where he joined Phelps Dodge Communications Company, Denmark Branch. He has worked in the areas of tropospheric propagation and antenna array theory. Recently he has been mainly interested in the theory of diffraction and its application to reflector antennas.

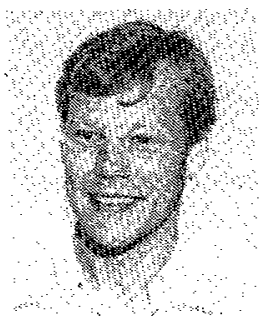

Hans-Henrik Viskum (S'84-M'85) was born in Copenhagen, Denmark, in 1957. He received the M.Sc.E.E. and Ph.D. degrees from the Technical University of Denmark in 1982 and 1985, respectively.

From 1982-1986 he was employed as a research associate at Electromagnetics Institute, TUD, working on reflector antenna analysis and synthesis, numerical methods and near-field measurements. In 1986 he joined the International Telecommunications Satellite Organization (INTELSAT), Washington, $\mathrm{DC}$, as a member of the technical staff in the Antennas and Propagation Section of the Research and Development Department. 